\title{
Preface to the special issue on geometric reasoning
}

\author{
Jacques Fleuriot ${ }^{1} \cdot$ Tetsuo Ida $^{2}$
}

Published online: 23 May 2015

(c) Springer International Publishing Switzerland 2015

While much of geometry was done with pencil and paper in the past, we are now fortunate to have the computer as an additional tool that can help us investigate its formal representation and semantics, and apply appropriate reasoning procedures. This special issue, with a selection of six papers that combine both theory and practice, demonstrates that geometry still has much to offer to our understanding of a wide array of concepts and ideas, whether through deductive or algebraic reasoning. We briefly review a few of the salient aspects of the various contributions next.

In Automated Generation of Machine Verifiable and Readable Proofs: A Case Study of Tarski's Geometry, Sana Djurdjevic, Julien Narboux and Predrag Janičić provide an interesting evolution of automated reasoning with respect to Tarski's geometry. They demonstrate that through a judicious choice of logic for representation and of interactive and automated tools for reasoning, it is possible to produce readable proofs for a significant number of theorems fully automatically. This is noteworthy since Tarski's axiomatics, developed as the basis for a decision procedure for elementary algebra and geometry, has often been viewed as too low-level to produce automatic proofs that humans would find conceptually interesting.

In the paper Formalizing Complex Plane Geometry, Filip Marić and Danijela Petrović describe a comprehensive mechanization of the extended complex plane and various fundamental concepts, such as Möbius transformations. The work in the proof assistant Isabelle/HOL deals with aspects that have not been mechanized previously and its

Jacques Fleuriot

jdf@inf.ed.ac.uk

Tetsuo Ida

ida@cs.tsukuba.ac.jp

1 School of Informatics, University of Edinburgh, Edinburgh, UK

2 University of Tsukuba, Tsukuba, Japan 
foundational nature suggests that it could act as a catalyst for further theorem proving formalization, e.g. in non-Euclidean geometries, by other researchers.

The paper Formalizing a Discrete Model of the Continuum in Coqfrom a Discrete Geometry Perspective by Nicolas Magaud, Agathe Chollet and Laurent Fuchs investigates discrete geometry in the proof assistant Coq. The work distinguishes itself through its nonstandard, yet constructive, approach that involves a model of the continuum over the Harthong-Reeb line. In particular, the authors construct a nonstandard model of integer arithmetic that enables the automatic extraction of computational contents from the proofs in Coq.

In the paper Automated Generation of Geometric Theorems from Images of Diagrams, Xiaoyu Chen, Dan Song and Dongming Wang describe a combination of mathematical and AI-based approaches from pattern-recognition, conjecture making and theorem proving aimed at discovering geometric theorems from digital images of diagrams. This work sets the foundations for discovering geometric concepts from various diagrammatic sources, including noisy ones, and may be valuable as part of a tool for teaching geometry, where hand-drawn diagrams, for instance, are given as input.

The paper A Singular web service for geometric computations by Francisco Botana and Zoltan Kovacs describes work on the design and implementation of a web-service around the computer algebra system Singular. This work is timely since a significant amount of recent research in automated reasoning aims at integrating tools running as web services in order to tackle classes of problems that were hitherto impossible. In this particular case, by taking advantage of the powerful polynomial manipulation capabilities of Singular, GeoGebra is enhanced to deal with algebraic loci and envelopes.

Finally, the paper Lang's Universal Molecule Algorithm by John C. Bowers and Ileana Streinu provides a deep analysis of Lang's Universal Molecule algorithm for 3D origami design. The work strives to clarify various computational aspects and provides a detailed proof of correctness of the reformulated algorithm.

We end this foreword with thanks to everyone who submitted a manuscript to this special issue and to the reviewers for their time and insightful comments.

Jacques Fleuriot Tetsuo Ida 